\title{
Thulium-fiber-laser-pumped, high-peak-power, picosecond, mid-infrared orientation-patterned GaAs optical parametric generator and amplifier
}

\author{
L. Xu*, Q. Fu, S. Liang, D.P. Shepherd, D.J. Richardson and S.-U. Alam \\ Optoelectronics Research Centre, University of Southampton, Southampton, SO17 1BJ \\ *Corresponding author: I.xu@soton.ac.uk
}

Received XX Month XXXX; revised XX Month, XXXX; accepted XX Month XXXX; posted XX Month XXXX (Doc. ID XXXXX); published XX Month XXXX

\begin{abstract}
We report a high-peak-power, picosecond, mid-infrared optical parametric generator (OPG) and amplifier (OPA) based on orientation-patterned GaAs (OP:GaAs) pumped by a Tm:fiber master-oscillator-power-amplifier (MOPA) employing direct diode-seeded amplification. An OPG tuning range of $2550-2940 \mathrm{~nm}$ (signal) and 5800-8300 $\mathrm{nm}$ (idler) is demonstrated with peak powers as high as 3 kW (signal) and $2 \mathrm{~kW}$ (idler), and with pulse energies of $0.26 \mu \mathrm{J}$ and $0.16 \mu \mathrm{J}$, respectively. When seeded with a 0.6 cm$^{-1}$ linewidth tunable Cr:ZnSe laser, the OPA idler linewidth is narrowed to $1.4 \mathrm{~cm}^{-1}$ and a small-signal parametric gain of $60 \mathrm{~dB}$ is achieved. A maximum peak power of $13.3 \mathrm{~kW}$ (signal) and $3.2 \mathrm{~kW}$ (idler) is obtained at an overall conversion efficiency of $36 \%$. The corresponding maximum pulse energies for signal and idler are $1.07 \mu \mathrm{J}$ and $0.26 \mu \mathrm{J}$, respectively. (C) 2017 Optical Society of America
\end{abstract}

OCIS codes: (190.4970) Parametric oscillators and amplifiers; (190.4400) Nonlinear optics, materials; (190.7110) Ultrafast nonlinear optics.

http://dx.doi.org/10.1364/OL.99.099999

Tunable sources of short laser pulses in the mid-IR (2 to $20 \mu \mathrm{m}$ ) have important applications in the area of chemical sensing and spectroscopy due to the presence of characteristic vibrational absorptions of a large number of molecules in this spectral region $[1,2]$. Orientation-patterned gallium arsenide (OP-GaAs) has become an attractive nonlinear crystal for this spectral region as it has large nonlinear coefficients, wide transparency, good thermal conductivity and the capability of designer quasi-phase-matching (QPM)[3]. However, owing to the onset of two-photon absorption, OP-GaAs can only be pumped at wavelengths above $1.7 \mu \mathrm{m}$ [4]. Thulium (Tm)-doped fiber lasers, working at around $2-\mu \mathrm{m}$ wavelength, have been considered a good candidate for directly pumping OP-GaAs to obtain mid-IR wavelengths by nonlinear frequency conversion, offering the possibility of compact sources with high efficiency. For example, an optical parametric oscillator
(OPO) pumped by a 100-ns pulsed Tm-fiber laser has generated output average power as high as $800 \mathrm{~mW}$ at around $10.6 \mu \mathrm{m}$ [5]. Whereas, the short range of tunability and the low peak power of the output $(\sim 160 \mathrm{~W})$ could potentially limit its application. Using a femtosecond $\mathrm{Tm}$ fiber laser, a tuning range covering 3- to 6- $\mu \mathrm{m}$ output was demonstrated from a synchronously pumped OPO [6]. The peak power was $\sim 8 \mathrm{~kW}$ at $3.8 \mu \mathrm{m}$, while it decreased to $640 \mathrm{~W}$ when the wavelength was tuned to $5.6 \mu \mathrm{m}$. However, strong limitations on the output average power $(\sim 10 \mathrm{~mW}$ in a singly resonant oscillator) were noted in this work due to the high pump intensities leading to three-photon absorption.

Compared to OPOs with complex cavities, optical parametric generators (OPGs) are very attractive, due to their simple singlepass nature, allowing compact devices that are less costly and less sensitive to external perturbations. OPGs can also be injected by external seed lasers to work as optical parametric amplifiers (OPAs), thereby combining the spectral and spatial (beam-quality) characteristics of the seed while taking advantage of the high gain that nonlinear frequency-conversion techniques offer. The first OPA in OP-GaAs was realized by seeding with a $4.5-\mu \mathrm{m}$ quantumcascade laser (QCL) and pumping with a Tm-fiber-laser-pumped Ho:YAG Q-switched laser [7]. In that work the OPA provided a gain of 53-dB and generated 30-ns pulses with a peak power of $580 \mathrm{~W}$. In later work, [8], a Tm-fiber-laser directly pumped OP-GaAs OPA was demonstrated with a tuning range from 8 to $10 \mu \mathrm{m}$ and output pulses with $\sim 30$ ns duration. However, the output peak power was reported to be less than $200 \mathrm{~W}$.

Here we describe a widely tunable OPG based on OP-GaAs that is directly pumped by a nearly all-fiber Tm:MOPA system operating at $1952 \mathrm{~nm}$, generating $\sim 80$ ps duration pulses at $1 \mathrm{MHz}$ repetition rate and employing simple, direct diode-seeded amplification. By injection-seeding with a narrow linewidth continuous-wave (CW) laser for OPA operation, both the output signal and idler spectral linewidth were significantly decreased and output powers as high as1.07 W and $0.26 \mathrm{~W}$, for signal $(2605 \mathrm{~nm})$ and idler $(7785 \mathrm{~nm})$ respectively, were obtained. The corresponding peak powers were $13.3 \mathrm{~kW}$ (signal) and $3.2 \mathrm{~kW}$ (idler) and the overall conversion efficiency was $36 \%$. To the best of our knowledge, these are the 
highest mid-IR peak powers reported from an OP-GaAs crystal. These results also represent the first report of an OP-GaAs OPG with average output powers higher than $100 \mathrm{~mW}$.

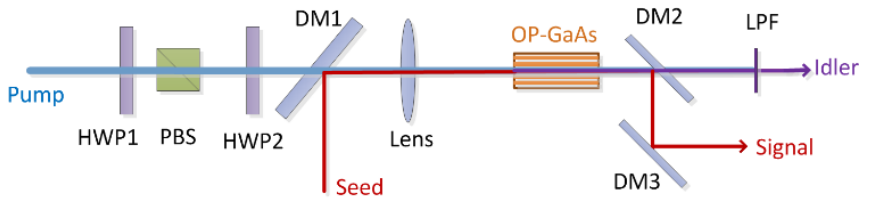

Fig. 1. Experimental setup of the OPG (unseeded) and OPA (seeded). HWP, half-wave plate; PBS, polarizing beam splitter; DM, dichroic mirror; LPF, long-pass filter.

The configuration of the OPG and OPA is shown in Fig. 1. The pump source was a diode-seeded Tm-doped-fiber MOPA system employing direct amplification, similar to that reported in [9]. It consisted of a PM-fiber-pigtailed gain-switched laser-diode seed laser, operating at a central wavelength of $1952 \mathrm{~nm}$ and a repetition rate of $1 \mathrm{MHz}$, followed by two core-pumped pre-amplifier stages. A fiber-pigtailed electro-optic modulator (EOM) was then used as a time gate to remove the excess amplified spontaneous emission (ASE) in between pulses. A grating-based filter consisting of a pair of fiber Bragg gratings (FBGs) was employed to narrow down the linewidth to $0.3 \mathrm{~nm}$. A third-stage amplifier with $11-\mu \mathrm{m}$-core fiber and fourth-stage amplifier with $25-\mu \mathrm{m}$-core fiber were used to suppress the nonlinearities and combined with cladding-pumping to increase the output power. The output pump beam at $1952 \mathrm{~nm}$ was measured to have a beam quality of $\mathrm{M}^{2}=1.3$. The power input to the OPG/OPA was varied using a half-wave plate placed before a polarizing beam splitter, as shown in Fig. 1. Another half-wave plate was then used to rotate the beam polarization orientation to realize maximal quasi-phase matching in an OP-GaAs crystal. The beam after the dichroic mirror (DM1, Fig. 1) was focused into the OP-GaAs by an uncoated $\mathrm{CaF}_{2}$ lens with a beam waist of $80 \mu \mathrm{m}\left(1 / \mathrm{e}^{2}\right.$ radius of intensity). The 20-mm-long OP-GaAs crystal (BAE Systems) had five gratings with periods ranging from $57 \mu \mathrm{m}$ to $65 \mu \mathrm{m}$ in steps of $3 \mu \mathrm{m}$. Each grating had a dimension of 1-mm thickness (aligned to the [001] crystallographic axis), 5-mm width (aligned to [110]) and 20 -mm length (aligned to [1 10$]$ ), and the pump beam propagated along the length of the crystal. The crystal was mounted in an oven to allow temperature tuning. Both end facets of the OP-GaAs were anti-reflection (AR) coated at the pump $(\mathrm{R}<1 \%)$ and signal $(\mathrm{R}<6 \%)$ wavelengths, but had a reflection of up to $17 \%$ at the longest idler wavelength $(8 \mu \mathrm{m})$. The relatively high loss at the idler wavelength was because the coating was originally designed for a different operating wavelength and could easily be reduced. The signal output was extracted using two dichroic mirrors (DM2, DM3, Fig. 1), with high transmission at the pump and idler wavelengths and high reflection at the signal wavelength. The idler power was measured after a long-pass filter (LPF, Fig. 1), which had a short wavelength cut-off at $4.5 \mu \mathrm{m}$. The signal and idler output powers discussed in the following text correspond to that immediately after the OP-GaAs crystal, taking into account the losses of the DMs and LPF but not the AR coatings on the crystal.

The available average power from the MOPA system, after passing through the various optical elements before the OP-GaAs crystal was $3.7 \mathrm{~W}$. The pump pulses had a duration of $\sim 80 \mathrm{ps}$ and a spectral linewidth of $0.5 \mathrm{~nm}$. The maximum intensity at the focal point in the OP-GaAs crystal was thus around $230 \mathrm{MW} / \mathrm{cm}^{2}$. Although it was well below the crystal damage threshold, any higher intensity would cause significant three-photon absorption effects which induces pump loss and lead to related thermal issues $[6,10]$. Without seed laser injection, the generation of signal and idler pulses was observed at a minimum pump threshold of around $1.6 \mathrm{~W}$, with the pump polarization set at an angle of $58^{\circ}$ with respect to the horizontal axis (i.e. at $58^{\circ}$ to [110]) in the single-pass OPG process.

To investigate the behavior and influence of the pump polarization on the three-wave mixing in the OP-GaAs, we measured the generated idler output power against the angle of the input pump polarization. It has previously been reported that the orientation of the crystallographic axes with respect to the polarization direction of the pump, signal and idler waves has a strong influence on the frequency conversion [5,11-13]. Theoretical models predict that the effective nonlinear coefficient has a maximum when the pump polarization is aligned to the [111] direction (35 to the [110]) [12]. However, as shown in Fig. 2, we observed a global maximum idler output power when pumping with the polarization at an angle of $58^{\circ}$ to [110]. Possible explanations for this discrepancy are the presence of stress or thermally induced birefringence in the OP-GaAs or the non-optimal optical coatings [5,13].

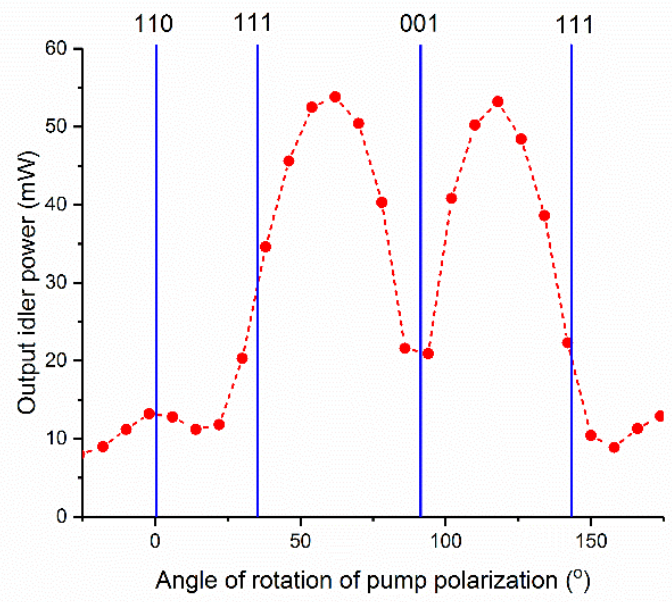

Fig. 2. Idler average output power versus the angle of pump polarization angle and the corresponding crystal axis.

Using a grating period of $57 \mu \mathrm{m}$ and an oven temperature of 50 ${ }^{\circ} \mathrm{C}$, signal at a wavelength of $2930 \mathrm{~nm}$ and idler at a wavelength of $5860 \mathrm{~nm}$ were generated from the OP-GaAs crystal with a maximum average power of $260 \mathrm{~mW}$ and $160 \mathrm{~mW}$, respectively, when pumped at $3.5 \mathrm{~W}$. Therefore, considering the total output power, the overall power conversion efficiency of the OPG was $12 \%$. With a $1 \mathrm{MHz}$ repetition rate, the corresponding pulse energies for signal and idler were $0.26 \mu \mathrm{J}$ and $0.16 \mu \mathrm{J}$, respectively. Unfortunately, the pulse duration of the signal and idler pulses could not be measured due to the lack of suitable equipment, but we would expect that they are equal to or slightly shorter than that of the pump in the OPG process [14]. In this case, the peak power of the generated signal and idler pulses are at least $3 \mathrm{~kW}$ and $2 \mathrm{~kW}$, respectively. By using different $\mathrm{OP}-\mathrm{GaAs}$ grating periods and oven temperatures, the signal (idler) wavelength could be tuned from $2550 \mathrm{~nm}$ to $2940 \mathrm{~nm}$ (5800 nm to $8300 \mathrm{~nm}$ ), as shown in Fig. 3. The multiple spectral modulated lines indicates the absorption of $\mathrm{H}_{2} \mathrm{O}$ in the air. The spectral linewidth were relatively broad at $20 \mathrm{~nm}(29$ 
$\left.\mathrm{cm}^{-1}\right)$ and $200 \mathrm{~nm}\left(33 \mathrm{~cm}^{-1}\right)$ for the signal and idler, as expected from an OPG. The calculated gain bandwidth, defined by the bandwidth at which the gain falls to one half of the maximum, is slightly smaller at $20 \mathrm{~cm}^{-1}$ for signal and idler [15], but the linewidth may be broadened due to effects such as grating errors, temperature variance along the crystal and focusing effects.
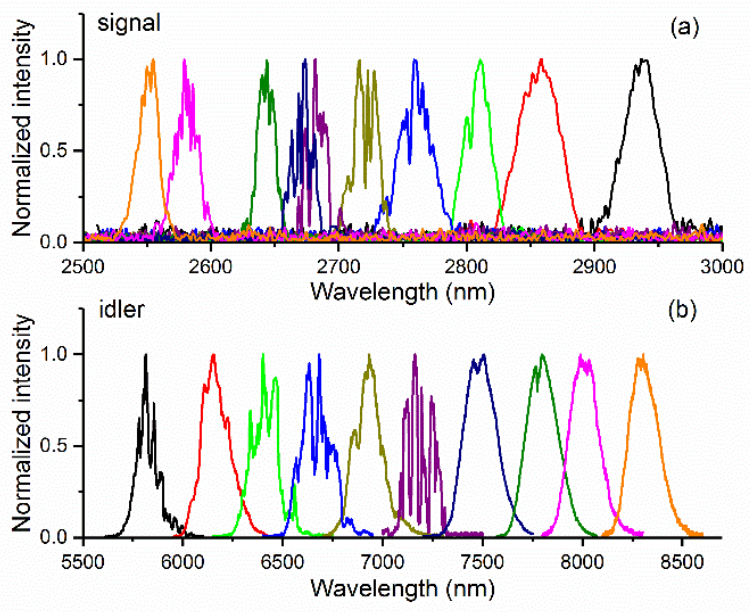

Fig. 3. Measured spectra for signal (a) and idler (b) from the tunable OPG.

Some applications, such as chemical sensing, require mid-IR laser sources with large spectral tunability but with narrow linewidth to achieve high spectral resolution [8]. In order to narrow the spectral linewidth, a commercial, continuous wave (CW), tunable Cr:ZnSe laser (IPG Ltd) was employed as an injection signal to seed the OPG for OPA operation. The seed laser had a linewidth of $0.6 \mathrm{~cm}^{-1}$ and wavelength tunability from $1.9 \mu \mathrm{m}$ to $2.7 \mu \mathrm{m}$. The seed and pump beams were combined by a dichroic mirror (DM1, Fig. 1). Due to the elliptical output beam of the seed laser, the beam waist after the focusing lens was $140 \mu \mathrm{m}$ by $80 \mu \mathrm{m}$ in the horizontal and vertical

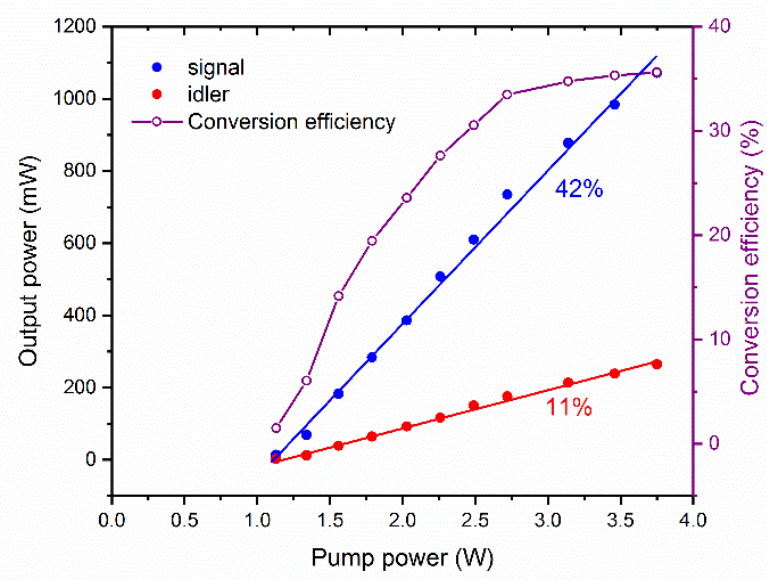

Fig. 4. (Left) Signal and idler output power as a function of pump power in OPA operation. Circles are measured data points and the solid lines are linear fits. (Right) Total power conversion efficiency versus pump power. The solid line is purely to guide the eye. planes, respectively. Thus, there was only one direction where the pump (waist size $80 \mu \mathrm{m}$ ) and signal beams had good overlap. As the seed beam was horizontally linearly polarized (aligned to [110]), the pump polarization was also chosen to be parallel to the seed to maximize the conversion efficiency [12]. With $280 \mathrm{~mW}$ of seed laser power at a wavelength of $2650 \mathrm{~nm}$, the pump threshold decreased to approximately $1 \mathrm{~W}$. Figure 4 shows that the output powers of the generated signal (CW seed excluded) and idler increased linearly with slopeefficiencies of $42 \%$ and $11 \%$, respectively. The maximum signal power of $1.07 \mathrm{~W}$ at $2650 \mathrm{~nm}$ and idler power of $0.26 \mathrm{~W}$ at $7440 \mathrm{~nm}$, were obtained at a pump power of $3.7 \mathrm{~W}$. The corresponding maximum overall power conversion efficiency was $36 \%$ and the effective total slope efficiency was $53 \%$. The maximum pulse energies for signal and idler were $1.07 \mu \mathrm{J}$ and $0.26 \mu \mathrm{J}$, respectively. Taking into account the duty cycle of the 80-ps pulses and the 1-MHz repetition rate, the CW seed power of $280 \mathrm{~mW}$ corresponds to an effective average seed power of only $22.4 \mu \mathrm{W}$ within the pulse duration. Thus the $1.07 \mathrm{~W}$ output represents a gain of $46 \mathrm{~dB}$.

Assuming the signal and idler had the same pulse durations as the pump, the attainable peak power was $13.3 \mathrm{~kW}$ and $3.2 \mathrm{~kW}$ for the signal and idler, respectively. As shown in Fig.5, the OPA signal linewidth was reduced to $0.5 \mathrm{~nm}$, while the idler linewidth was narrowed to $8.5 \mathrm{~nm}$. As a result, the OPA leads to more than 20 times increase in spectral density in comparison to the OPG. As expected, the frequency bandwidth was essentially transferred from the pump $\left(1.3 \mathrm{~cm}^{-1}\right)$ to the idler $\left(1.4 \mathrm{~cm}^{-1}\right)$, with the narrower seed bandwidth $\left(0.56 \mathrm{~cm}^{-1}\right)$ being transferred to the amplified signal $\left(0.71 \mathrm{~cm}^{-1}\right)[16]$. By tuning the seed wavelength, a tunable output signal from $2557 \mathrm{~nm}$ to $2684 \mathrm{~nm}$ (limited by the tunability of the seed laser) and a tunable idler from $7168 \mathrm{~nm}$ to $8267 \mathrm{~nm}$ were achieved from the OP-GaAs OPA by using different grating periods and temperatures. Therefore, the OPA has generated tunable signal and idler picosecond pulses with narrow linewidth and high peak power, which could also be potentially suitable for techniques such as mid-IR differential absorption LIDAR [17].

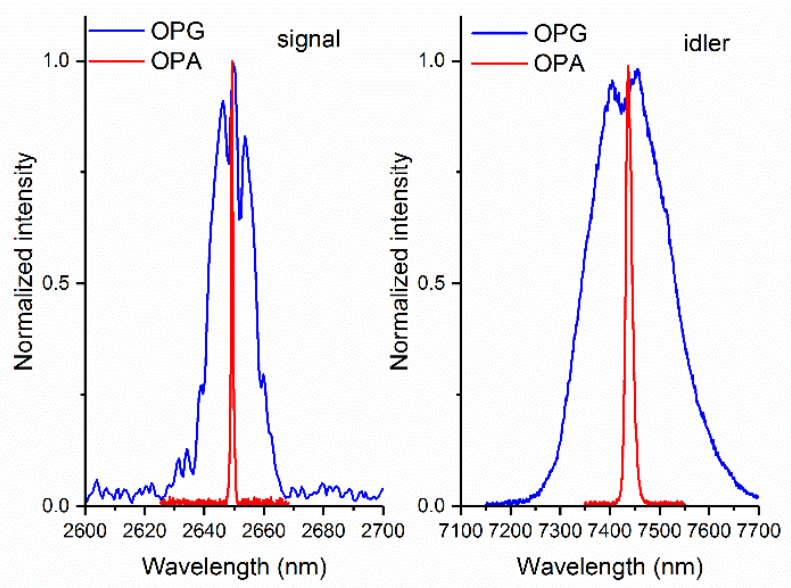

Fig. 5. Measured spectra for signal (left) and idler (right) in both OPG and OPA operation regime.

The signal and idler beam quality were characterized in both OPG and OPA mode by using a Pyrocam-based profiler (NanoScan, Photon). In OPG mode the signal beam quality was measured to be $\mathrm{M}^{2}{ }_{\mathrm{x}}=1.7$ and $\mathrm{M}_{\mathrm{y}}{ }_{\mathrm{y}}=1.8$, and the idler beam quality was $\mathrm{M}^{2} \mathrm{x}=2.3$ and 
$\mathrm{M}_{\mathrm{y}}^{2}=2.4$ in the horizontal and vertical planes, respectively. In OPA mode the signal beam quality was measured to be $\mathrm{M}^{2} \mathrm{x}=2.8$ and $\mathrm{M}^{2} \mathrm{y}$ $=2.7$, which was degraded compared to the OPG. This could be attributed to the poor overlap of the circular pump beam and elliptical seed beam, as mentioned previously. However, the idler beam quality was measured to be $^{2}{ }_{\mathrm{x}}=1.9$ and $\mathrm{M}_{\mathrm{y}}^{2}=1.8$, which was improved in comparison to the OPG.

An exploration of the requirement and influence of the seed laser power on the parametric gain in the single-pass OPA process was performed. With the pump power fixed at $3 \mathrm{~W}$, we measured the signal output power by seeding the OPA at different power levels. As illustrated in Fig. 6, the extractable power from the OPA becomes saturated as the seeding effective average power exceeds $10 \mu \mathrm{W}$. For smaller seed powers gains as high as $60 \mathrm{~dB}$ were observed.

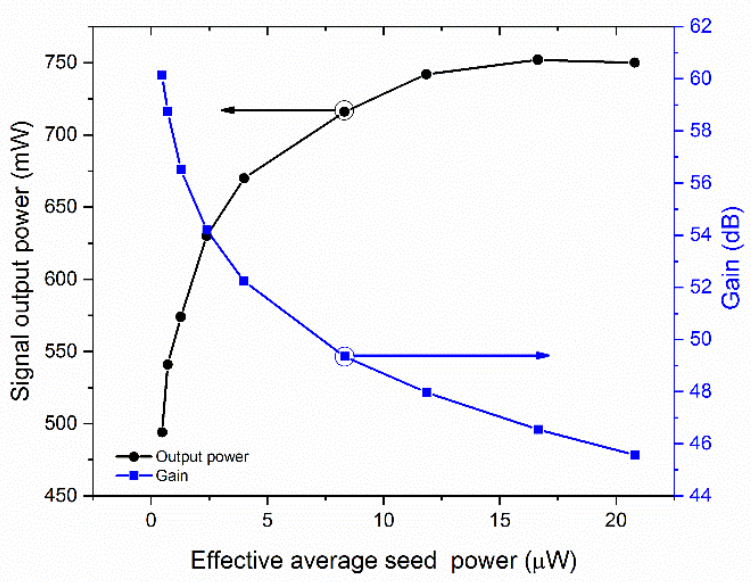

Fig. 6. Signal output power and Gain as a function of different seeding power in OPA.

It should be noted that the maximum pump intensities used in these picosecond frequency-conversion experiments $\quad$ (230 $\mathrm{MW} / \mathrm{cm}^{-2}$ ) were much lower than those required in the femtosecond OPO experiments described in [6], as much longer OPGaAs crystal lengths could be used. Thus, three-photon absorption effects were avoided (we calculate maximum losses of $4 \%$ in our experiments), allowing efficient operation with high average and peak powers.

In conclusion, we have demonstrated a high-peak-power, widely tunable, picosecond optical parametric generator (OPG) based on OP-GaAs pumped by a gain-switched-diode-seeded Tm-dopedfiber MOPA system employing direct amplification. The OPG has generated signal and idler with wavelength tuning ranges of 2550 $2940 \mathrm{~nm}$ and $5800-8300 \mathrm{~nm}$, with pulse peak powers (energies) of $3 \mathrm{~kW}(0.26 \mu)$ and $2 \mathrm{~kW}(0.16 \mu)$ ), respectively. Seeded with a 0.6 $\mathrm{cm}^{-1}$ linewidth continuous-wave tunable Cr:ZnSe laser, the idler linewidth was narrowed to $1.4 \mathrm{~cm}^{-1}$ in single-pass optical parametric amplifier (OPA) operation and a small signal parametric gain of $60 \mathrm{~dB}$ was achieved. Maximum peak powers of $13.3 \mathrm{~kW}$ (signal) and $3.2 \mathrm{~kW}$ (idler) were obtained at an overall conversion efficiency of $35 \%$. The corresponding maximum pulse energies for signal and idler were $1.07 \mu \mathrm{J}$ and $0.26 \mu \mathrm{J}$, respectively.

Funding. Engineering and Physical Science Research Council (EPSRC) Grant EP/I01196X/1 and EP/I02798X/1. The data for this paper can be found at doi.org/10.5258/SOTON/D0220.
Acknowledgment. We thank Dr. Peter Schunemann from BAE Systems for growing the OP-GaAs crystals and thank Prof. Anna Peacock from ORC, University of Southampton for providing the tunable seed laser for our work.

\section{References}

1. F. K. Tittel, D. Richter, and A. Fried, "Mid-Infrared Laser Applications in Spectroscopy," in Solid-State Mid-Infrared Laser Sources, I. T. Sorokina and K. L. Vodopyanov, eds. (Springer Berlin Heidelberg, Berlin, Heidelberg, 2003), pp. 458-529.

2. M. W. Sigrist, J. Adv Res 6, 529-533 (2015).

3. T. Skauli, K. L. Vodopyanov, T. J. Pinguet, A. Schober, O. Levi, L. A. Eyres, M. M. Fejer, J. S. Harris, B. Gerard, L. Becouarn, E. Lallier, and G. Arisholm, Opt. Lett. 27, 628-630 (2002).

4. P. G. Schunemann, in Advanced Solid State Lasers, OSA Technical Digest (online) (Optical Society of America, 2015), AM2A.2.

5. J. Wueppen, S. Nyga, B. Jungbluth, and D. Hoffmann, Opt. Lett. 41, 42254228 (2016).

6. O. H. Heckl, B. J. Bjork, G. Winkler, P. Bryan Changala, B. Spaun, G. Porat, T. Q. Bui, K. F. Lee, J. Jiang, M. E. Fermann, P. G. Schunemann, and J. Ye, Opt. Lett. 41, 5405-5408 (2016).

7. G. Bloom, A. Grisard, E. Lallier, C. Larat, M. Carras, and X. Marcadet, Opt. Lett. 35, 505-507 (2010).

8. F. Gutty, A. Grisard, C. Larat, D. Papillon, M. Schwarz, B. Gérard, R. Ostendorf, J. Wagner, and E. Lallier, Adv. Opt. Techn. 6, 95 (2017).

9. A. M. Heidt, Z. Li, J. Sahu, P. C. Shardlow, M. Becker, M. Rothhardt, M. Ibsen, R. Phelan, B. Kelly, S. U. Alam, and D. J. Richardson, Opt. Lett. 38, 1615-1617 (2013).

10. W. C. Hurlbut, Y.-S. Lee, K. L. Vodopyanov, P. S. Kuo, and M. M. Fejer, Opt. Lett. 32, 668-670 (2007).

11. K. L. Vodopyanov, O. Levi, P. S. Kuo, T. J. Pinguet, J. S. Harris, M. M. Fejer, B. Gerard, L. Becouarn, and E. Lallier, Opt. Lett. 29, 1912-1914 (2004).

12. P. S. Kuo, K. L. Vodopyanov, M. M. Fejer, X. Yu, J. S. Harris, D. F. Bliss, and D. Weyburne, Opt. Lett. 32, 2735-2737 (2007).

13. C. Kieleck, M. Eichhorn, A. Hirth, D. Faye, and E. Lallier, Opt. Lett. 34 262-264 (2009).

14. L. Xu, H.-Y. Chan, S.-U. Alam, D. J. Richardson, and D. P. Shepherd, Opt. Express 23, 12613-12618 (2015).

15. G. D. Boyd and D. A. Kleinman, J. Appl Phys 39, 3597-3639 (1968).

16. J. Prawiharjo, H. S. S. Hung, D. C. Hanna, and D. P. Shepherd, J. Opt. Soc. Am. B 24, 895-905 (2007).

17. P. Geiser, U. Willer, D. Walter, and W. Schade, Appl Phys B 83, 175 (2006). 


\section{References with full titles}

1. F. K. Tittel, D. Richter, and A. Fried, "Mid-Infrared Laser Applications in Spectroscopy," in Solid-State Mid-Infrared Laser Sources, I. T. Sorokina and K. L. Vodopyanov, eds. (Springer Berlin Heidelberg, Berlin, Heidelberg, 2003), pp. 458-529.

2. M. W. Sigrist, "Mid-infrared laser-spectroscopic sensing of chemical species," J. Adv Res. 6(3), 529-533 (2015).

3. T. Skauli, K. L. Vodopyanov, T. J. Pinguet, A. Schober, O. Levi, L. A. Eyres, M. M. Fejer, J. S. Harris, B. Gerard, L. Becouarn, E. Lallier, and G. Arisholm, "Measurement of the nonlinear coefficient of orientation-patterned GaAs and demonstration of highly efficient second-harmonic generation," Opt. Lett. 27(8), 628-630 (2002).

4. P. G. Schunemann, "New Nonlinear Optical Crystals for the MidInfrared," in Advanced Solid State Lasers (Optical Society of America, Berlin, 2015), p. AM2A.2.

5. J. Wueppen, S. Nyga, B. Jungbluth, and D. Hoffmann, "1.95 $\mu \mathrm{m}$ pumped OP-GaAs optical parametric oscillator with $10.6 \mu \mathrm{m}$ idler wavelength," Opt. Lett. 41(18), 4225-4228 (2016).

6. O. H. Heckl, B. J. Bjork, G. Winkler, P. Bryan Changala, B. Spaun, G. Porat, T. Q. Bui, K. F. Lee, J. Jiang, M. E. Fermann, P. G. Schunemann, and J. Ye, "Three-photon absorption in optical parametric oscillators based on OP-GaAs," Opt. Lett. 41(22), 5405-5408 (2016).

7. G. Bloom, A. Grisard, E. Lallier, C. Larat, M. Carras, and X. Marcadet, "Optical parametric amplification of a distributed-feedback quantum-cascade laser in orientation-patterned GaAs," Opt. Lett. 35(4), 505-507 (2010).

8. F. Gutty, A. Grisard, C. Larat, D. Papillon, M. Schwarz, B. Gérard, R. Ostendorf, J. Wagner, and E. Lallier, "High peak-power laser system tuneable from 8 to $10 \mu \mathrm{m}$," in Advanced Optical Technologies, 6, 95 (2017).

9. A. M. Heidt, Z. Li, J. Sahu, P. C. Shardlow, M. Becker, M. Rothhardt, M. Ibsen, R. Phelan, B. Kelly, S. U. Alam, and D. J. Richardson, "100kW peak power picosecond thulium-doped fiber amplifier system seeded by a gain-switched diode laser at $2 \mathrm{~mm}$," Opt. Lett. 38(10), 1615-1617 (2013).

10. W. C. Hurlbut, Y.-S. Lee, K. L. Vodopyanov, P. S. Kuo, and M. M. Fejer, "Multiphoton absorption and nonlinear refraction of GaAs in the mid-infrared," Opt. Lett. 32(6), 668-670 (2007).

11. K. L. Vodopyanov, O. Levi, P. S. Kuo, T. J. Pinguet, J. S. Harris, M. M. Fejer, B. Gerard, L. Becouarn, and E. Lallier, "Optical parametric oscillation in quasi-phase-matched GaAs," Opt. Lett. 29(16), 19121914 (2004).

12. P. S. Kuo, K. L. Vodopyanov, M. M. Fejer, X. Yu, J. S. Harris, D. F. Bliss, and D. Weyburne, "GaAs optical parametric oscillator with circularly polarized and depolarized pump," Opt. Lett. 32(18), 2735-2737 (2007).

13. C. Kieleck, M. Eichhorn, A. Hirth, D. Faye, and E. Lallier, "Highefficiency 20-50 kHz mid-infrared orientation-patterned GaAs optical parametric oscillator pumped by a $2 \mu \mathrm{m}$ holmium laser," Opt. Lett. 34(3), 262-264 (2009).

14. L. Xu, H.-Y. Chan, S.-U. Alam, D. J. Richardson, and D. P. Shepherd, "High-energy, near- and mid-IR picosecond pulses generated by a fiber-MOPA-pumped optical parametric generator and amplifier," Opt. Express. 23(10), 12613-12618 (2015).

15. G. D. Boyd, and D. A. Kleinman, "Parametric interaction of focused Gaussian light beams," Journal of Applied Physics. 39(3597 (1968).

16. J. Prawiharjo, H. S. S. Hung, D. C. Hanna, and D. P. Shepherd, "Theoretical and numerical investigations of parametric transfer via difference-frequency generation for indirect mid-infrared pulse shaping," J. Opt. Soc. Am. B. 24(4), 895-905 (2007).

17. P. Geiser, U. Willer, D. Walter, and W. Schade, "A subnanosecond pulsed laser-source for mid-infrared LIDAR," Appl Phys B. 83(2), 175 (2006). 